\title{
FIVE DIMENSIONS OF ONLINE PERSONA
}

\author{
Christopher Moore, Kim Barbour, and Katja LeE
}

\begin{abstract}
Before Facebook, Twitter, and most of the digital media platforms that now form routine parts of our online lives, Jay Bolter (2000) anticipated that online activities would reshape how we understand and produce identity: a 'networked self', he noted, 'is displacing Cartesian printed self as a cultural paradigm' (2000, p. 26). The twenty-first century has not only produced a proliferation and mass popularisation of platforms for the production of public digital identities, but also an explosion of scholarship investigating the relationship between such identities and technology. These approaches have mainly focussed on the relations between humans and their networks of other human connections, often neglecting the broader implications of what personas are and might be, and ignoring the rise of the non-human as part of social networks. In this introductory essay, we seek to both trace the work done so far to explore subjectivity and the public presentation of the self via networked technologies, and contribute to these expanding accounts by providing a brief overview of what we consider to be five important dimensions of an online persona. In the following, we identify and explicate the five dimensions of persona as public, mediatised, performative, collective and having intentional value and, while we acknowledge that these dimensions are not exhaustive or complete, they are certainly primary.
\end{abstract}

\section{KEY NODES OF RESEARCH}

The scope of research in this field is wide and varied, fruitfully informed by multiple disciplinary perspectives. Here we trace only a handful of scholars and concepts, focusing in particular on work that is foundational or influential in our formulations of the dimensions of online persona. Harrison Rainie and Barry Wellman (2012), for example, advance the notion of 'networked individualism', which helps to acknowledge and account for the connections between online activity and the formation of subjectivity. They remind us that communication technologies, media platforms, and digital services are not isolated objects or discrete entities, but are voraciously incorporated into the lives of individuals as part of the extant identity assemblage that is undergoing continuous revision, updates, and patching as we form connections and exchange information with other people and other systems. Zizi Papacharissi's (2010) media and communication perspective presents us with another elaboration of the 'networked self', a term which she uses to indicate the construction of a subjective performance across multiple and simultaneous streams of social awareness that expands autonomy, potentially reduces agency, and which requires constant self-surveillance and monitoring.

Philosophers Alexander Galloway and Eugene Thacker (2007), have expressed a 'nostalgia' for a time when there was no need to produce quantitative data about the self but, drawing on the work of Alfred North Whitehead, Mark Hansen (2015) argues that within any performance of subjectivity-human or nonhuman-there is a generalised subjectivity that inheres within quantitative data; a "dispersal of agency across networks" $(2015$, p. 3$)$ that is a marker of the elemental character of contemporary media. According to Hansen, Whitehead's speculative philosophy, along with his insistence on the universality of subjectivity as the basis for a re-anchoring of human experience within media networks that have become substantially decoupled from direct human perception, helps us to appreciate the irreducible sensory dimension of the "data-fied" experience:

...subjectivity acquires its power not because it incorporates and processes what is outside, but rather through its direct co-participation or sharing in the 
polyvalent agency of myriad subjectivities. Our distinctly human subjectivity is the result of a complex assemblage of overlapping, scalevariant microsubjectivities functioning distinctly and autonomously" (Hansen 2015, p. 12).

Hansen draws on Whitehead's speculative approach and metaphysical scheme to substantiate the understanding of the neutrality of subjective qualities as being powerfully valuable in theorising the everyday experience of digital, networked and social media.

Other key researchers, such as Nancy Baym, have examined how interpersonal media forms of communication accelerate new constitutions of "personal connection" (2010, p. 1). Baym's work on digital identity draws on Donath's (2007) useful notion of signalling, which works to locate social position within an information-saturated society. Using Facebook and it's templates as a case study, Laurie McNeill's work (2012) has explored the collaboration between non-human and human components in producing online autobiographical acts. Anna Poletti and Julie Rak (2014) offer a similar orientation of a networked identity within biographical and autobiographical studies in Identity Technologies: Constructing the Self online, an edited collection that argues such technologies are a fundamental part of the online world in the contemporary era. These writers and thinkers, among others, have advocated novel means for considering the construction of identity within a technologically diverse social order. They have begun to examine how the individual is intimately connected to the presentation of their public selves within online culture, through digital connections to social institutions, and via the networked organisations of everyday life that are fundamentally different to what has come before.

Absent from these discussions, however, is persona and yet persona is both the product of and interface for the movement of the individual into online activities. As the first editorial for this journal noted (Marshall and Barbour 2015), it is these very activities that have yielded the contemporary 'proliferation of personas for both presentation and strategic purposes' on a massive scale (2015, p. 1): 'persona-making as a practice', we noted, has become 'pandemic' $(2015$, p.9). Across the various contributions to this journal since then contributors have readily taken up persona as a critical lens through which to understand identity practices and performances in online contexts. The five dimensions of online persona explored here-the public, the mediatised, the performative, the collective, and intentional value-build upon that work and, we contend, form a productive means for understanding the configuration of online identity in the contemporary era.

\section{The Public Dimension of Online Persona}

Publicness is the first dimension of contemporary online personas. While there still exists a lingering sense of early-to-mid 1990s utopian 'net' philosophy with its libertarian discourse about anonymity and freedom afforded by early internet technologies like multi-user experiences and web forums, much of the obscurity between a user's online presentation and offline selves has been obliterated over the past decade. The eradication of anonymity has been achieved by the 'real name' requirements and end user license agreements of online game services, social media terms of service contracts, and the ubiquitous presence of browser-based tracking cookies. This trend has been strengthened with government-sanctioned and metadata enabled state surveillance, resulting in an online experience that is almost always public in some way. The user of a web-enabled service-from wearable technologies like a Fitbit, or an app-based experience which incorporates a Google search query, or public API interaction-is almost always an extension into a wider public. Rainie and Wellman (2012) describe networked individuals at the centre of what Marshall (2013), and Barbour et al. (2014) have labelled as 'micro-publics', the extended social network of the individual that includes personal friends, professional associates, plus their networks, and the systems and platforms that connect them 
all. This approach advocates for taking into consideration all the technologies, devices, apps, API's and the physical as well as digital infrastructure that encapsulates contemporary public networked identity.

This first dimension of an online persona is comprised of a particularly wide-ranging spectrum of 'publicness' and at each point along its traversal exists the very real potential to go from a small public of close and intimate friends to a massive and global public audience, enabled by the act of sharing. This potentiality parallels the historical notion of the production of the public self such as when celebrities and stars start out performing to small public audience, but later attain a larger audience as they become more popular and well known. This trajectory gives us insights into the dynamics of online persona creation, which always anticipates this shift from small to larger scale publics.

In this industrial model of the individual, the public self is the 'official' version that the celebrity offers up to the world, a highly polished, scheduled and controlled version that is produced and performed for launches, premieres, speaking engagements, and other live and mediated promotions, appearances, and events. High-profile celebrities and public figures have teams of publicity assistants and staff that work to maintain consistency in this public persona because, as Turner, Bonner, and Marshall (2000) note, the celebrities (and their personas, we would add) are "commodities, produced to be marketed in their own right or to be used to market other commodities. The celebrity's ultimate power is to sell the commodity that is themselves" (2000, p. 12). Organisations, brands, institutions and commercial entities similarly have this public-facing dimension of their online persona with teams of social management operatives conducting licensed online persona management, and a range of employees with quasi-official public selves connected to these identities. Celebrities, brands, and organisations are all especially important public figures because of their pedagogical functions, as they help us to identify new aspects of agency and risk. In the past, the media gatekeeping of celebrity would have relegated figures like Kim Kardashian to tabloid notoriety, but the degree of agency provided by control over the public presentation of the self online has meant a global celebrity built on careful management from her initial public notice (through the leaking of her 'sex tape') into a fashion, music, marketing, and promotional career. As Marshall (2014) suggests celebrities act as pedagogical markers providing replicable frameworks for the conduct of the public presentation of the self. This mediatised identity is organised through multiple commercial applications, networks and platforms, which is not only shared to a personal public by the individual, but becomes a source for information harvesting, advertising, and massive commercial sharing (Marshall 2015).

\section{Mediatised Dimension of Persona}

The mediatised dimension of persona follows on from the first dimension of publicness, as an expression of the self. This is not a new phenomenon: individuals have been mediatising themselves via communication technologies in perpetuity from rock painting, portraits, journals and letters, to ham radio call signs, autobiographies, and social media profiles. The contemporary assemblage of persona now combines multiple media technologies: even a simple selfie requires mobile screens, cameras, digital image compression algorithms, and communication across wireless or telecommunication carrier signals, APIs, and hashtags. Billions of daily social media users, across Facebook, WeChat, Twitter, and Instagram demonstrate an unparalleled scope of skills and degree of comfortability with public mediatisation and express unprecedented levels of actual and potential public exposure (see Marshall 2015; 2016; 2017). Mediated persona operates under the modulation of commercial interest and corporately governed structures that individuals have become acclimatised to, many in the hope of sharing in the benefits of a widely proliferating self-image that was once only the province of individuals in film, television, print, and radio. The codes of conduct and the practices of the mediated public often conflict, but they also mirror the conservative values of 
the once dominant 'press,' such as Facebook and Instagram's ban on the female nipple. What is new in this mediatisation of the self, however, is the naturalisation of platform censorship and the negotiation between the personal, corporate, and institutional agency.

Celebrity is an important pedagogical firmament in the relationship of individuals to their extra-textual dimensions of mediatised public identity, which is, as Marshall (2017) reflects, part of the primary work of the actor. Whether stage actress, radio performer, television presenter, news anchor, or Hollywood A-lister, actors undergo an incessant and structured construction and presentation of the self for the purpose of promotion well beyond that of the theatre, show, or movie text being offered for consumption. For many actors, the work of professional performance and the products of marketing and advertising become paratextual components to their public identity (see Naremore 1991, Dyer 2004). The many iconic roles of actors like Tom Cruise, Harrison Ford, Scarlett Johansson, or Charlize Theron, for example, are also paratextual components of their star celebrity persona. Developed from the narratological theory of Gerard Genette (1997), paratexts are liminal devices or conventions (like red-carpet poses, characteristic facial attributes, Instagram habits, or Facebook pages) that form a threshold of meaning between text and audience. Genette describes paratexts as heterogeneous practices and discourses acting as thresholds between author, publisher, and audience. The mediatised identities of online persona are formed by the accumulation of paratexts over time; appearances at film festivals and award ceremonies, for example, are an important paratextual mediatisation of celebrity identity. These paratexts circulate as performers use their identities to proselytise themselves and the productions they are part of, becoming visible as a mediatised figure, via traditional media distribution channels and newer and more personal social media platforms. Affect is naturalised in this mediatisation of the self through individualised platform paratexts including likes, favourites, shares and retweets. Furthermore we are witness to the massive proliferation of dedicated channels for the remediation of traditional broadcast media paratexts (editing methods, generic conventions, and other mise-en-scene) that are formed through meta-mediatisation via platforms such as YouTube and TwitchTV.

\section{Performative Dimension of Persona}

Just as the mediatisation follows on from the public elements of online persona, performativity, the third dimension is also essential requirement and extension of the first two. To present a publicly mediated persona, we must perform our identity, our profession, our gender, and effectuate our tastes, interests, and networks of connection, through activities like commenting on posts, liking other's contributions or framing a selfie. This performative identity does not make claims about the 'real', or a self that is somehow less produced or implemented, or more complete in some underlying way. The public performance of the self is neither entirely 'real' nor entirely 'fictional'. The accomplishment of performativity means that a persona connects together and meshes all the various characteristics that are staged and presented in the everyday and intended to interact with others.

Erving Goffman $(1959,1971)$ documented what can be understood as the recurring patterns of performativity and accounted for the methods of impression formation and management within a dramaturgical model of the presentation of the self that differentiated between the public 'front stage' and the private 'backstage'. Goffman conveyed an understanding of the degree to which we all present 'faces' and act outwardly depending on any given situation and its expectations. The performance of self varies between the identifiable roles, from parent to employee, friend to a colleague, teacher to student, and none are any more 'real' or 'fictional' than the others. As Papacharissi (2010) observed, any interactions between a performed self and the performed self of others, can quickly become a pattern of action which then becomes routine, creating and then normalising a narrative of expected behaviour in any given situation: in an earlier editorial Lee (2015) explored this pattern for persona performance 
in work contexts. We come to rely on this patterning of interaction, both offline and online, in order to help regulate and make predictable our networks of interactions: we understand that images of family shared via Facebook are going to be liked by the extended networks of friends and familiar relations, while news stories relevant to our given professions will be liked by colleagues and peers, and that there may be some overlap occurring between the two groups depending on proximity, familiarity, and so on. Social media and mobile technologies are now old enough for us to understand that different generations of users have developed different approaches to the performativity of the self that may appear to blur the front and back stages, but this merely reminds us that the methods of performing the self are not fixed (see danah boyd 2010).

Judith Butler's (1999) approach to gender extended the notion of identity performativity and our understanding of the degree to which core elements of our public self are constructed for and by us. Butler's model contests the idea that gender is 'natural', 'real' or 'innate' to a fiction or a projection that is applied to and by individuals who are fundamentally negotiating their public selves through an articulation of power. She argued that the presentational quality of (gendered) identity is neither biologically determined nor individually produced, but rather both enabled and constrained by the institutions, technologies, networks, and cultures in which the public self is assembled and performed. Performativity has also been considered in Speech Act theory (see Austin 1975, Berns 2014 (whose earlier work influenced Bulter), Marshall 2017), which considers speaking as a component of identity formation. The performance of speech-whether verbal, physical, textual or otherwise-is a part of the movement of identity in action and interpretation, and this theoretical framework has been featured in ethnographic and anthropological discussions of identity (Berns 2014.).

The Habermasian interpretation of the 'lifeworld' (1987) is also productive as a means of understanding the performance of the public self online. For Habermas, the lifeworld embodies the symbolic reproduction of society, thus the lifeworld of an online identity is one comprised of media platforms, mobile technologies, multiple communication channels, and modes of behaviour. To perform the lifeworld is to wrangle these elements together, to manage the self across a diverse range of structures, institutions, technical performances, frames, and stages. This doesn't necessarily need to be a complicated exercise of online habitus: Ken Hillis points to the simplified performance and naturalised ideology of the "pull-down menu" (2009, p. 29), which has become so normalised in our everyday performances of the self when we sign up to a new app, social media service, or online video game world. Hillis reminds us the performance of gender, height, age, profession, location, attitude, and relationship to others is purely ritualised as result of the limited options available to users in the system (Hillis 2009). The performative dimension of persona marks a high degree of agency in the public presentation of the self online, but this agency is inevitably contested. As Marshall notes, it is through the performativity of presentational media that individuals are encouraged, invoked, and even "seduced" into more elaborate constructions of public presentation", and they are "drawn into a performativity that operates as a continuous marketing of the self's value" (Marshall, 2010, np). The acts of performing the self online are so diverse that an individual may pick and choose the aspects of the role that best suits their intended performance (Barbour 2015), but this performance is a balancing act between multiple registers ranging from the personal and intimate to the public and professional, and must be carefully articulated to remain sincere and authentic.

\section{Collective Dimension of Persona}

The fourth dimension of persona is one that works to produce, seek out, and move between connections, resulting in a collective. This dimension is observed across all forms of social media (see boyd \& Ellison 2007) as persona is mediated and then publicly distributed across the connections and networks that users manage via services and platforms. 
Participation in these online networks results in multiple publics that are significantly different from the public associated with traditional broadcast media or political association. No longer is the individual 'part' of a collective, but rather the individual is connected to multiple publics, making the collective dimension of persona a meta-collective complex. In each public, the individual is a node, but they are also simultaneously orbiting nodes in other networks. The complex overlapping of networks, however, can still be thought of as having a central point, which is the user's persona.

This networking of activity from friends and followers across all these intercommunicating networks can be described as a 'micro-public' (Marshall 2014; Barbour et al. 2014). Similar to the notion of a 'personal public' (Schmidt 2013), the concept of a micropublic is one that takes into account the practices of social media such as sharing, tagging, and mediated expression in the forms of personal images, memes, likes, and dislikes. The intercommunication between micro-public activities occur as part of the interpersonal communication of the self, where self-mediations are linked to self-promotional activity across multiple platforms, sites, and services (Marshall 2015). Take, for example, the eponymous selfie, which is framed using an internet-connected device and distributed via multiple platforms each with their own, possibly overlapping audience. Instagram is designed with this purpose in mind, offering the user the capacity to send the image to Facebook, Twitter, Flickr and other services at the point of self-publication. Micro-publics have a tremendous porousness, connecting to other networks effortlessly and with often unpredictable and unforeseen consequences.

The concept of micro-public is crucial to persona studies as a core means for describing the collective dimensions of online persona and the ways that groups, associations, and networks have become central to contemporary cultures. Micro-public formations are explored in various other interactions by researchers including Theresa Senft (2008), Alice Marwick (2013) and Alice Marwick and danah boyd (2011), where the term serves as a means to highlight a new duality. Personas can tap into a potentially massive audience and can feature tens, hundreds, thousands and even millions of individual followers, who are all nodes in a massively personal network. Micro-publics are micro, not in terms of scale, but with regards to the nature of the network that is regularly and privately updated by a central identity. A micropublic is attached to a unique persona that is personally producing, responding, and broadcasting in the tradition of previously dominant media institutions, which makes the micropublic a quasi-public network. To grapple fully with the emergence of the online persona, we look closely at the strong connections between individuals and the multiple overlapping micropublics to which they are central. Twitter is an important platform for micro-public formation. For example, Twitter is heavily relied on by journalists to develop followings and build affective connections to listeners, viewers, and readers who may never visit or consume their home publications. Similarly, the public personas of such journalists are not like the standardised cutouts of representational culture, but living and breathing presentational figures who have direct and often unfiltered connections their audiences. The dynamic of the audience is complicated by the friending and following relationship that amplifies the affective bond between author and audience (or celebrity and fan, politician and voter, and so on) that has contributed new interpersonal dimensions to cultural expression, governance, and consumption. We can see this emerge in what Marshall (2014) describes as the intercommunication industries which service micro-publics, both massively large ones achieved by popular artists and those smaller but equally vibrant and successful ones set up by more independent operators who are still central to their own networks and maintain modest, vibrant, and active followings. Professional social media sites such as LinkedIn and Academia.edu are examples of platforms which service the operation of micro-public formation and management. Public persona emerges from and across these presentational media forms and their micro-publics intercommunicatively, forcing a renewed focus on the management of reputation (Barbour \& Marshall 2012). The very complex construction of publics as micropublics intersects with larger and well-established media and communication systems that 
produce powerful cultural tropes, which contribute to a new orientation of value and agency which can be seen in the last dimension of online persona.

\section{Value Dimension of Persona}

The final dimension of persona that we discuss here relates to the idea of value, and how that value is dependent on agency, reputation, and prestige. Collectively known as VARP (Marshall 2016), this dimension recognises that personas are created with a particular intention. The intent to create personas can vary from the personal or intimate (designed to facilitate personal or familial relationships) to the professional (more associated with work), or the public (produced by those who wish to claim a level of fame or notoriety). Personas are not fixed to the original intention which led to their creation, but rather slip between registers of performance (Barbour 2014), a process that is facilitated by the mediated, collective nature of persona production.

In that recognition of the intent behind persona production is encoded an understanding of the agency involved. Although working within the affordances and constraints of technology, power structures, and social and cultural norms, those producing personas are still making active and important decisions in how they perform that persona to their micro-publics. The mask of persona is adopted through its performance, and the persona can then become a 'thing' through which other 'things' can be achieved. The production of networks happens through the actions of the producer(s) of the persona, and members of those networks might equally contribute to that persona through their choices and actions. Para-textual actions such as 'liking', or sharing particular content, are active contributions to a public or quasi-public identity, and demonstrate the importance of the choices we make when engaging online.

How we understand the value of the personas we produce can influence how we understand the significance of the reputation those personas maintain. Specific characteristics may be emphasised in online spaces to produce a particular type of reputation, and research conducted into the aspirational nature of online identity performances (Yurchison, Watchravesringkan \& McCabe 2005; Zhao, Grasmuck \& Martin 2008; Whittkower 2014) appears to support the idea that aspirational characteristics are often eventually subsumed and incorporated into an offline persona, even if they were initially exaggerated online. Although we do not see online personas as necessarily 'fake', this could be understood through the adage of 'fake it 'til you make it'.

The prestige associated with the persona is significant in that it draws together, and is reliant on, the previous elements of this dimension (value, agency, reputation), as well as all the other dimensions of persona explicated above. The prestige associated with personas - as understood by those who create them - is bestowed by the persona's micro-public, and the fact that prestige itself relies on widespread positive affirmation means that, in this case, having a larger micro-public to provide that affirmation would certainly influence how prestigious a persona could be considered to be (or consider themselves to be). However, as with all elements of online persona creation, size of micro-public is relative: producing an active, engaging persona for an admiring extended family group may be felt as prestigious for one person, while another may be dissatisfied with an enthusiastic Twitter following numbering in the hundreds of thousands. Here, we return to ideas of agency and intent: when the intent is to connect with certain others through a persona, the value of that persona will be measured by its ability to achieve that aim. 


\section{IN THIS ISSUE}

Through this journal, we have continued our original project to engage with "comprehending, analysing, and critiquing persona" (Marshall \& Barbour 2015), and in this open issue, the papers attend to this project in distinct and fascinating ways.

In '"Get Off My Internets": How Anti-Fans Deconstruct Lifestyle Bloggers' Authenticity Work,' Sarah McRae examines how communities of 'anti-fans' coalesce in online forums around different lifestyle bloggers. These communities, McRae's research demonstrates, are particularly attentive to the persona performances of the bloggers, and actively monitor and evaluate how authenticity is performed according to ever shifting constructions of legitimacy.

In 'The persona in autobiographical game-making as a playful performance of the self,' Stefan Werning introduces us to the niche but growing genre of autobiographical video games. These games, his work suggests, make quite literal the playful possibilities of persona construction and performance within the constraints and affordances of the technologies and platforms. Such playfulness extends to both game-makers, who design an iteration of self that can be occupied, amended, and played, and for gamers who take up and play or inhabit these personas.

Patrick Osborne's 'Constructing the Antichrist as Superstar: Marilyn Manson and the Mechanics of Eschatological Narrative' focuses on construction of Manson's controversial persona as an 'Antichrist' in the 1990s and the crucial role that Christian organisations and ideological frameworks played in not only crafting that persona, but infusing it with meaning and value. The persuasiveness and popularity of Manson's persona, Osbourne suggests, owes much to that backlash.

In 'The Hyphenated Persona: Aidan Quinn's Irish-American Performances,' Loretta Goff guides us through how 'Irish-Americanness' has been performed across the twentieth century, and how it continues to be performed in American contexts today. Using Aidan Quinn as a case study, Goff's work emphases not only the flexibility and the multiplicity of persona performances, but also their strategic commercial and affective value when redeployed in the appropriate venues to certain publics.

The creative practice contribution to this issue comes from Anastasia Salter and Bridget Blodgett. Their work of hypertext literature - part social media experience, part game, part choose your own adventure - embeds the reader/player in the US Twittersphere during the final months of 2016 and January 2017. The work explores the everyday struggles of a Twitter user in this time, challenging and discomforting the reader/player as choices in how to engage with the online space trigger responses in the form of changing follower counts as well as interactions with colleagues and strangers. In inhabiting the persona of a Twitter user at this specific moment in recent history, the artists challenge us to think about how social media persona creation impacts on, and is impacted by, other embodied presentational practices.

Across this issue, we can trace a recurring interest in the role of networked publics in the construction of the persona. While not all of these contributions are concerned specifically with the formation and function of such networks and connections in online contexts, all are attentive to the thresholds marking persona, performance, and publics, and, importantly, the dynamic interplay between these constituents. Such play is quite literal in both Werning's work and Salter and Blodgett's creative work which highlight how publics are, in certain digital platforms, not only encouraged to interact with personas but play constitutive roles in persona production and performance. We are reminded by these works of the potential porousness of the thresholds that mark and distinguish personas and publics, and the richness of experience and play that is possible on such platforms. Goff's work on the mobility of hyphenated personas 
and the multiplicity of publics that make and take meaning from such persona performances is a useful reminder as well that publics are not just porous but overlapping. Moreover, the persona that transverses such publics is a dynamic, mobile, and inherently flexible construction capable of performing and signalling differently as needed.

Such mobility is, in Quinn's case, an asset with clear commercial value but in the case of lifestyle bloggers, McRae's work points out, such flexibility and inconsistency can be a liability. The community of 'anti-fans' that McRae investigates are highly critical of contradiction and code inconsistent performances as hypocrisy and inauthenticity. Yet, because the mechanisms for signalling authenticity are always on the move, the lifestyle blogger must continually adapt and shift their performances. As with the Christian publics who took issue with Manson, the performative nature of persona and its capacity to operate as neither 'real' nor 'fiction,' is ofttimes flattened out by critical publics oriented by an agenda to evaluate and oppose. In Manson's case to make his persona performance 'real' gives life to these publics, and a tangible, measurable, stable 'threat' against which they can work; in the case of lifestyle bloggers, the anti-fans arbitrate what are real and what are faked performances. At the heart of both, is not only a desire for a correspondence between performance and 'real world' identity, but also accountability. To hold Manson or lifestyle bloggers accountable for their performances is to acknowledge their agency and strategy, but it is also a practice that, at least in these cases, is predicated upon publics marking firm thresholds between persona and public, assuming an oppositional stance in that network, and presuming an authority that is often moral or moralising in its execution. As both Osborne and McRae demonstrate, such practices might easily overlook the complexities of the networks that bind personas and publics, and, in particular, the capacity of the publics to play roles in persona production and performances.

When we are mindful of these collectives and networks and the play that happens within and through them, and attentive to the performances, the mediations, and the mechanisms of acquiring and distributing value through persona, as the articles in this issue are, it is inevitable that we consider as well the structural and structuring components that condition and constrain the person production and performance. In his examination of autobiographical games, Werning is attentive to how the technological constraints of the game platforms and game-maker designed limitations (in behaviour, discourse, and so on) give structure to the play. McRae's work looks to the structuring role of genre in not only persona performance but also, crucially, how publics orient themselves and respond to such performances. In both Goff and Osborne's work, the structures are ideological and are not only capable of summoning publics into networks of relations with personas, but, in some cases, providing particular and rigid scripts that condition the interplay between them.

The structures that condition persona are crucial considerations but they can, if we are not careful, rapidly overwhelm or overdetermine how we make sense of personas. We are now, perhaps, habituated to discussions of the constraint and affordances of technology and social media platforms in the production of public digital identities. In this article, we have proposed five clear dimensions of online persona that can be useful in assisting with the analysis of the presentation of the public self. While we acknowledge these dimensions are not exhaustive, we argue for their usefulness as a way of considering relationships between technology and public digital identities.

\section{WORKS CITED}

Austin, JL 1975, How to Do Things with Words, Harvard University Press, Cambridge.

Barbour, K 2014, Finding the Edge: Online persona creation by fringe artists, Doctoral Thesis, Deakin University, Australia. 
-2015 'Registers of Performance: Negotiating the Professional, Personal and Intimate in Online Persona Creation' in H Savigny, E Thorsen, D Jackson, \& J Alexander (eds.) Media, Margins and Popular Culture, Palgrave Macmillan, UK, pp. 57 - 69.

Barbour, K \& Marshall, PD 2012, 'The academic online: Constructing persona through the World Wide Web', First Monday, vol. 17, no. 9, retrieved 18 August 2012, <http://firstmonday.org/ojs/index.php/fm/article/view/3969>.

Barbour, K, Marshall, PD \& Moore, C 2014, 'Persona to Persona Studies', M/C Journal, vol. 17, no. 3 , retrieved 24 February 2015 , <http://journal.mediaculture.org.au/index.php/mcjournal/article/view/841>.

Baym, NK 2010 Personal Connections in the Digital Age, Polity, Cambridge.

Berns, U 2014, 'Performitivity' in P Hühn et al. (eds.), The living handbook of narratology, Hamburg University, Hamburg, retrieved 7 November 2016, <http://www.lhn.unihamburg.de/article/performativity>.

Bolter, J 2000, 'Identity', in T Swiss (ed.) Unspun: Key Concepts for Understanding the World Wide Web, New York University Press, New York, pp. 17 - 29.

boyd, d \& Ellison, N 2007, 'Social Network Sites: Definition, History, and Scholarship', Journal of Computer-Mediated Communication, vol. 13, no. 1, pp. 210-230, doi:10.1111/j.10836101.2007 .00393$.

Butler, J 1999, Gender Trouble, 2nd edn, Taylor and Francis, Hoboken.

Donath, J (2007) 'Signals in social supernets', Journal of Computer Mediated Communication, vol. 13 , no. 1, pp. 231-251.

Dyer, R 2004, Heavenly bodies: Film Stars and Society, Routledge, New York.

Habermas, J 1987 Theory of Communicative Action, Volume 2: Life-World and System: A Critique of Functionalist Reason, translated by Thomas McCarthy, Beacon Press, Boston.

Hansen, MB 2015, Feed-forward: On the Future of Twenty-first-century Media, University of Chicago Press, Chicago.

Hillis, K 2009, Online a lot of the time: Ritual, Fetish, Sign, Duke University Press, Durham.

Galloway, AR and Thacker, E 2007, The exploit: A theory of networks (Vol. 21), University of Minnesota Press, Minnesota.

Genette, G 1997, Paratexts: Thresholds of Interpretation, Cambridge University Press, Melbourne.

Goffman, E 1959, The presentation of self in everyday life, Anchor Books, New York.

Goffman, E 1971, 'Remedial interchanges', in E Goffman Relations in public: Microstudies of the public order, Basic Books, New York, pp. 95 - 187.

Lee, K 2015, 'Personas at Work', Persona Studies, vol. 1, no. 2, pp 1 - 13.

Marshall, PD 2010, 'Persona Studies: Mapping the Proliferation of the Public Self', International Conference on Celebrity and News, 13-15 September, Geneva, Switzerland.

- 2013, 'Persona studies: Mapping the proliferation of the public self', Journalism, vol. 15, no. 2, pp. 153-170, doi:10.1177/1464884913488720.

- 2014, Celebrity and power: fame in contemporary culture, (2nd ed.), University of Minnesota Press, Minneapolis.

- 2015, 'Monitoring Persona: mediatized identity and the edited public self', Frame: Journal of Literary Studies, vol. 28, no. 1, pp. 115-133.

- 2016, 'Exposure: the Public Self Explored', in PD Marshall \& S Redmond (eds), A Companion to Celebrity, Wiley Blackwell, Boston, pp. 497-517.

- 2017 [forthcoming] 'Visible Women and Men: branding, persona and the construction of quality in Australian film actors', in F Collins, S Bye, and J Landman (eds.), A Companion to Australian Cinema, Wiley-Blackwell, Boston.

Marshall, PD \& Barbour, K 2015, 'Making Intellectual Room for Persona Studies: a New Consciousness and a Shifted Perspective', Persona Studies, vol. 1, no. 1, pp. 1-12.

Marwick, AE 2013, Status Update: Celebrity, Publicity, and Branding in the Social Media Age, Yale University Press, New Haven. 
Marwick, AE \& boyd, d 2011, 'I tweet honestly, I tweet passionately: Twitter users, context collapse, and the imagined audience', New Media \& Society, vol. 13, no. 1, pp. 114-133, doi:10.1177/1461444810365313.

McNeill, L 2012, "There is no "I" in network: social networking sites and posthuman auto/biography', Biography, vol. 35, no. 1, pp. 65-82, doi: https://doi.org/10.1353/bio.2012.0009.

Naremore, J 1991, 'The performance frame', in J Butler (ed) Star Texts: Image and Performance in Film and Television, Wayne State University Press, Michigan, pp 102 - 114.

Papacharissi, Z 2010, A networked self: identity, community and culture on social network sites, Routledge, New York.

Poletti, A \& Rak, J 2014, Identity technologies: Constructing the Self Online, University of Wisconsin Press, Madison.

Rainie, L \& Wellman, B 2012, Networked: The New Social Operating System, The MIT Press, Cambridge, Massachusetts.

Schmidt, J-H 2013, 'Practices of Networked Identity', in J Hartley, J Burgess \& A Bruns (eds), A Companion to New Media Dynamics, Wiley, Chichester, pp. 365-374.

Senft, T 2008, Camgirls: Celebrity \& Community in the Age of Social Networks, Peter Lang, New York.

Turner, G, Bonner, F \& Marshall PD 2000, Fame Games: The Production of Celebrity in Australia, Cambridge University Press, Cambridge.

Wittkower, DE 2014, 'Facebook and dramauthentic identity: A post-Goffmanian theory of identity performance on SNS', First Monday, vol. 19, no. 4, retrieved 12 May 2017, <http://www.ojphi.org/ojs/index.php/fm/article/view/4858/3875>.

Yurchisin, J, Watchravesringkan, K \& Brown McCabe, D 2005, 'An Exploration of Identity ReCreation in the Context of Internet Dating', Social Behavior \& Personality: An International Journal, vol. 33, no. 8, pp. 735-750.

Zhao, S, Grasmuck, S \& Martin, J 2008, 'Identity construction on Facebook: Digital empowerment in anchored relationships', Computers in Human Behavior, vol. 24, no. 5, pp. 1816-1836, doi:10.1016/j.chb.2008.02.012. 